\title{
Towards Programmable Address Spaces
}

\author{
Andrew Gozillon ${ }^{\dagger}$ and Paul Keir* \\ University of the West of Scotland \\ High St., Paisley PA1 2BE, Scotland, United Kingdom \\ Email: *andrew.gozillon@uws.ac.uk, †paul.keir@uws.ac.uk
}

\begin{abstract}
High-performance computing increasingly makes use of heterogeneous many-core parallelism. Individual processor cores within such systems are radically simpler than their predecessors; and tasks previously the responsibility of hardware, are delegated to software. Rather than use a cache, fast on-chip memory, is exposed through a handful of address space annotations; associating pointers with discrete sections of memory, within trivially distinct programming languages. Our work aims to improve the programmability of address spaces by exposing new functionality within the LLVM compiler, and then the existing template metaprogramming system of $\mathrm{C}++$. This is achieved firstly via a new LLVM attribute, ext_address_space which facilitates integration with the non-type template parameters of $\mathrm{C}++$. We also present a type traits API which encapsulates the address space annotations, to allow execution on both conventional and extended $\mathrm{C}++$ compilers; and illustrate its applicability to OpenCL 2.x.
\end{abstract}

\section{INTRODUCTION}

$\mathbf{T}$ HE MAJORITY of today's architectures are heterogeneous. This means they contain at least two different types of processors or different local memory units. A familiar example of this is the modern personal computer (PC) which contains both a graphics processing unit (GPU) and a central processing unit (CPU). These architectures have immense potential as the extra processors tend to be specialized for particular tasks. For example, GPUs are specialized for rendering graphics. However, the parallel structure of the GPU lends it incredibly well to single instruction multiple data (SIMD) tasks on large data sets. This is commonly referred to as general-purpose computing on graphics processing units (GPGPU). Due to this several programming models centered on taking advantage of this aspect have been created. The two most iconic are OpenCL [1] and CUDA [2]. GPUs are exceptional at performing SIMD tasks, so much so that several of the supercomputers in the TOP500 list [3] use them.

However, power often comes at a cost. In the case of heterogeneous architectures, the complexity of the program increases for software aiming to take full advantage of the power available. One of the more common added complexities is memory management. Memory management is an important aspect of several heterogeneous architectures, as auxiliary processors of these architectures can have separate memory from their primary processor. One such architecture are PCs containing GPUs. Each GPU has dedicated memory and data must explicitly be transferred across from main memory by the CPU. Current GPGPU programming models also segment GPU memory into several distinct address spaces with different properties that help increase throughput.

\author{
Figure $1 \mathrm{C}++$ Address Space API \\ add_as_t $<$ int, $42>\mathrm{i}=12345$; \\ static_assert (get_as_v<decltype ( i ) $>==42)$; \\ assert $(\mathrm{i}==12345)$
}

In some cases, named address space qualifiers have been introduced to help associate variables with certain address spaces and thus certain properties. Named address space began in the Embedded C Extension [4], a set of optional extensions to the $\mathrm{C}$ programming language for use with embedded processors and have since spread. In fact, several GPGPU programming models make use of them for example CUDA, OpenCL and Metal [5]. An example from OpenCL is the _private qualifier which restricts the scope of a variable to a thread. Other programming models such as OpenACC [6] aim to promote a higher level view of GPGPU programming and abstract away address spaces from the programmer.

Both $\mathrm{C}$ and $\mathrm{C}++$ are commonly used or extended in GPGPU programming. In fact all of the above mentioned programming models and languages with address spaces use or extend $\mathrm{C} / \mathrm{C}++$ in some way to achieve their goal. However, despite the number of models that make use of both $\mathrm{C} / \mathrm{C}++$ and address spaces, there is no standard compiler or library support for address spaces within $\mathrm{C} / \mathrm{C}++$. We believe that a $\mathrm{C}++$ librarybased approach could assist greatly in bringing address space support to $\mathrm{C}++$. An API that new programming models could explore and integrate with would help improve portability and programmabillity. Having an API like this available also removes the requirement to extend the compiler for named address space support. As such we have created an $\mathrm{C}++$ template API that takes inspiration from the $\mathrm{C}++$ standard libraries type traits, an example can be found in Figure 1.

To aid in showcasing our API's viability we have decided to use the Clang [7] compiler's address space implementation. The Clang compiler's address space implementation is different from named address spaces. It takes the generic approach of accepting an integral parameter, provided by an end-user, to specify the value's address space, instead of having a fixed name set while building the compiler itself. This lends itself well to our API, which proposes integral parameters to index address spaces. Modifications to the LLVM compiler were made to add support for $\mathrm{C}++$ non-type template parameters; since submitted as a patch. Note however that the API works 


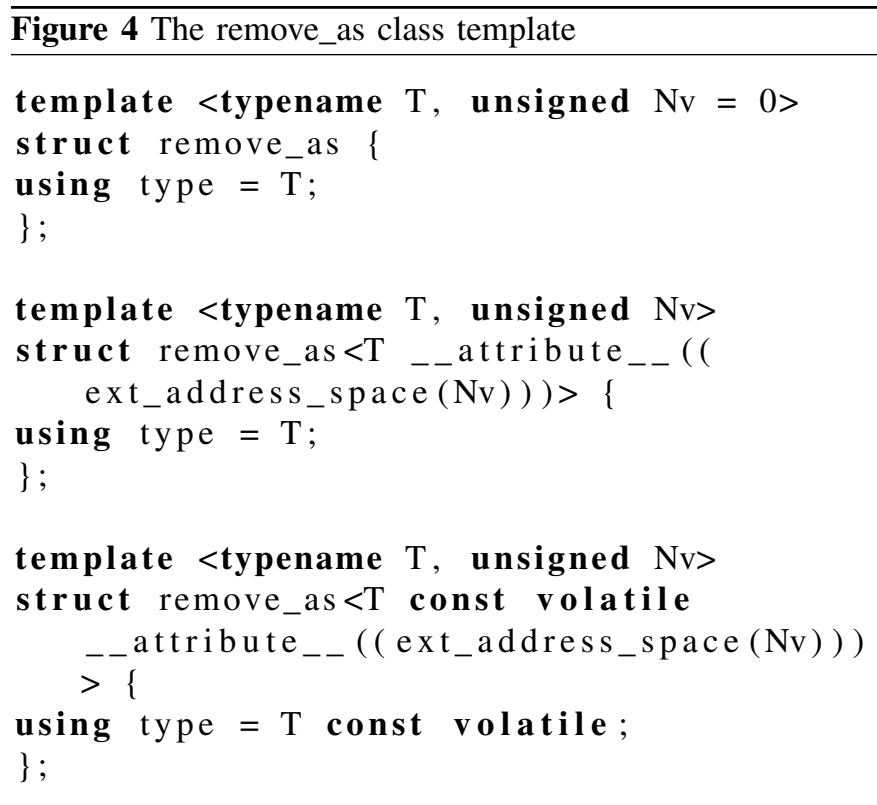

\section{B. The Traits API with as_val}

Each API function has a fall-back version for compilers that do not support the ext_address_space extension. This means that the API will not cause compilation errors or undefined behaviour, ensuring portability. Outwardly the API calls do not change, nor do the required includes. Only the implementation of the functions change significantly. This is handled by macros that check if the ext_address_space attribute is implemented, then includes the relevant header files.

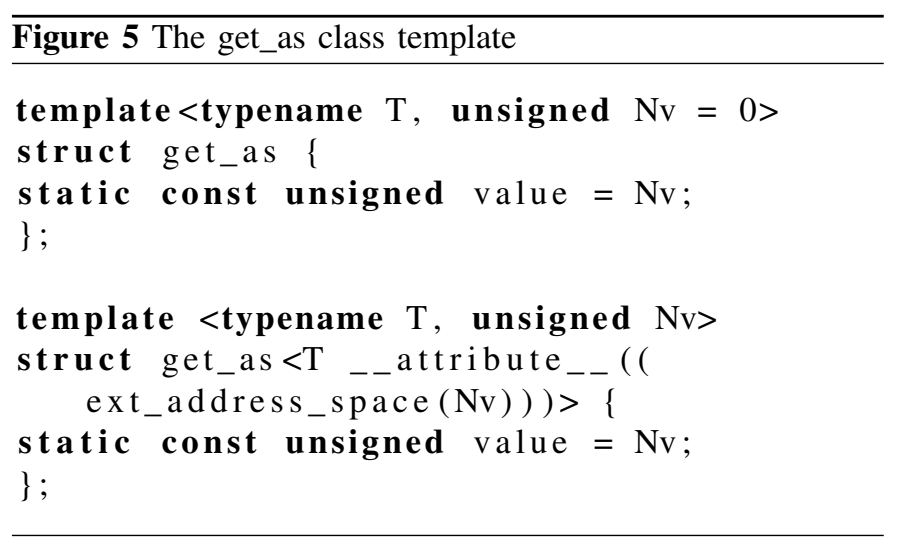

A template class facilitates the API implementation's mimicking of the address space qualifier. The as_val class template (Figure 6) accepts a template type parameter and two non-type template parameters. The type parameter represents the type the address space qualifier is to be applied to. The two nontype template parameters represent the address space of the top most pointer (the Nv parameter) and the address space of the pointee (the Np parameter). For example, as_val would only support address space qualifiers on the first two pointers of a pointer to a pointer to an integer type. The integer itself would not be qualifiable. Of the parameters, only the type is stored, the two address space values are stored at a type level and can be deduced. Through $\mathrm{C}++$ implicit conversion the various overloaded functions allow the user to use assignment operators and dereference operators as if they were using the base type. This means there should be no discernible difference between using a normal pointer type and as_val.

Whilst the implementations of the templates are different using the as_val class template. The change is not drastic. The functions all take in another non-type template parameter for the pointee address space (Np). However, this is hidden from the programmer using type aliases for each template.

The get_as implementation changes very little. Instead of deducing the value from the ext_address_space attribute currently tied to the type. It deduces the address space from the Nv parameter of the as_val class template.

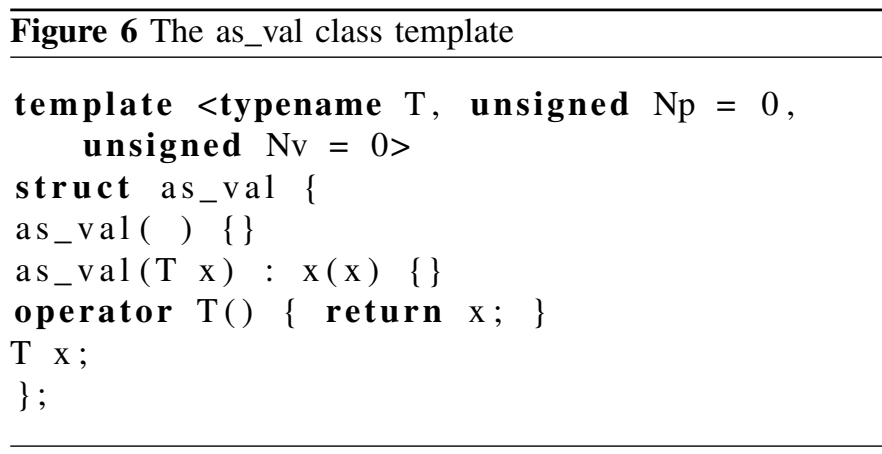

The implementation of remove_as is simplified. Instead of having multiple specializations for every qualifier combination. We can simply specialize for as_val. There is however a base case and specialization as we cannot simply return the type with the as_val (address space) removed. As there is also a pointee address space tied to as_val. The base case checks for an as_val with an 0 address space and pointee address space and returns as_val's stored type. The specialization checks for values greater than 0 in the Np parameter through deduction; then returns an as_val type with an Nv parameter set to 0 whilst keeping the same type and $\mathrm{Np}$ parameter.

For add_as we require a base template for non-as_val types and a specialization for types with as_val's. The base template wraps the given type with an as_val and sets the as_val types address space parameter to the given address space. Whereas the specialization simply replaces the current address space parameter of the as_val type with the newly given address space.

\section{The add_pointee_as and remove_pointee_as} Traits

From the description of these templates it's possible to notice that there is no way to set the pointee address space of an as_val template class. As such there are another two template classes that allow manipulation of the pointee. They are add_pointee_as and remove_pointee_as.

For the ext_address_space attribute extension the add_pointee_as template class requires base classes and specializations similar to the remove_as template. They also 
provide a similar use, to peel off const and volatile qualifiers from the passed in type and then reapply them to the return type. The template parameters and type alias in this case are also identical to add_as. The next step is to peel off the top level pointer to get access to its pointee (if it has one) and then apply an address space to it. This is achieved by a helper template which breaks down the type using the $\mathrm{C}++$ standard libraries pointer_traits template class and then rebuilding it. It does this in three steps, first it uses pointer_traits element_type parameter to get the pointees type. Secondly it applies the address space to this type. It finally uses pointer_traits rebind to bind the new type to the old type. The final result will be a type with a new address space on the pointee of the original pointer. For the non-extension implementation this is again much simpler. It is identical to add_as, except the pointee address space parameter is set instead.

\section{EXAMPLE USE CASE}

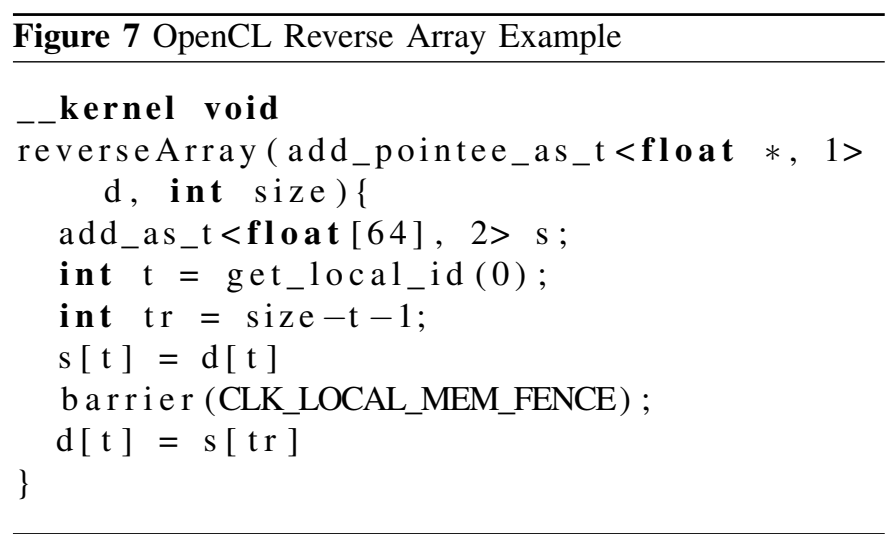

The example we chose to showcase the $\mathrm{C}++$ API can be found in Figure 7. The example is an OpenCL kernel function that will reverse one dimensional array data passed into it. The kernel function is based off a CUDA shared memory example found on NVIDIA's developer blog [8]. The idea is that each thread within a block will run an instance of this kernel and swap the relevant value based on its thread id. In this example the OpenCL named address spaces have been traded out for API calls which represent them as integer values. In the context of this example global is represented by the value one, local by the value two and private by the lack of a qualifier.

In the example the kernel accepts a pointer to some floating point data $\mathrm{d}$. Alongside an integer size that represents the number of elements contained within $d$. The float pointer type is wrapped in an add_pointee_as_t class template from our API with an integer representing the global address space. This applies the address space to the target of the float pointer. Which makes the type equivalent to _ global float*. Further down we create a statically allocated array of floats $\mathrm{s}$ which we can store values from $d$ in for swapping later. We apply add_as_t to its type alongside an integer representing the local address space. Which makes the type of $s$ equivalent to flocal float [64]. The function then creates two index values $t$ and $t r$ which represent the values we wish to swap within the current thread. After which we use the $t$ index to copy a value per thread from $\mathrm{d}$ in global memory to $s$ in local memory. However, before we can swap the data we must place a local barrier to prevent data races. After the barrier we can proceed to reverse the array.

A feature of our API is that if the attribute extension for ext_address_space is not found in the compiler it will still compile. It will fall back on the implementations that make use of the as_val template class which stores the address space value and variable within itself. Despite the variable now being wrapped within as_val it can still be used as if it was the raw type. This works through overloading certain operators in the class so that implicit conversion allows access to the underlying data. This fall-back functionality is provided by including all the required API functions through a single include file. This include file can then add different API implementations based on the presence of the ext_address_space attribute. This functionality has been tested with the GCC and Clang C++ compilers. Despite choosing OpenCL as the language for the example, the C++ API should be usable in the same way for $\mathrm{C}++$ and $\mathrm{C}++$ based programming models.

\section{CONCLUSiON}

In conclusion, we have presented a $\mathrm{C}++$ template API that we believe improves the programmability of address spaces. The API borrows concepts from C++'s type traits. We believe this API will help facilitate bringing address space qualifiers further into the $\mathrm{C}++$ type system allowing further template metaprogramming and type safety opportunities. To help showcase the ability of our API to integrate existing address space implementations, we integrated it with Clang's address space attribute. However we also presented an API implementation that would also allow it work as a standalone implementation of address spaces that requires no compiler extensions.

\section{REFERENCES}

[1] A. Munshi, "The opencl specification," in Hot Chips 21 Symposium (HCS), 2009 IEEE. IEEE, 2009. doi: 10.1109/HOTCHIPS.2009.7478342 pp. 1-314. [Online]. Available: http://dx.doi.org/10.1109/HOTCHIPS. 2009.7478342

[2] C. Nvidia, "Compute unified device architecture programming guide," 2007.

[3] T. S. Sites, “Top500 lists," 1993. [Online]. Available: https://www.top500. org/

[4] JTC1/SC22/WG14, "Programming languages - c - extensions to support embedded processors," 2006. [Online]. Available: http: //www.open-std.org/jtc1/sc22/wg14/

[5] Apple, "Metal," 2014. [Online]. Available: https://developer.apple.com/ metal/

[6] S. Wienke, P. Springer, C. Terboven, and D. an Mey, "Openacc first experiences with real-world applications," in European Conference on Parallel Processing. Springer, 2012. doi: 10.1007/978-3-64232820-6_85 pp. 859-870. [Online]. Available: http://dx.doi.org/10.1007/ 978-3-642-32820-6_85

[7] "clang: a c language family frontend for llvm." [Online]. Available: http://clang.llvm.org/

[8] M. Harris, "Using shared memory in cuda c/c++," 2013. [Online]. Available: https://devblogs.nvidia.com/parallelforall/ using-shared-memory-cuda-cc/ 